\title{
Improving the lateral resolution in confocal fluorescence microscopy using laterally interfering excitation beams
}

\author{
Olivier Haeberlé and Bertrand Simon \\ Laboratoire MIPS - Groupe Lab.El, Université de Haute-Alsace \\ IUT Mulhouse, 61 rue A. Camus F-68093 Mulhouse Cedex France \\ olivier.haeberle@uha.fr bertrand.simon@uha.fr
}

The confocal fluorescence microscope is the instrument of choice for biologists. However, compared to other instruments, its resolution is still limited. We propose a simple technique, based on laterally interfering beams, to improve the resolution. One technique consists in using a halve phase plate to modify the illumination, combined with a laterally offset detection. A $90 \mathrm{~nm}$ lateral resolution is obtained for properly prepared specimens using readily available dyes. Another approach is to use several excitation beams, slightly shifted and properly dephased, to decrease the lateral extension of the PSF. With this approach, a lateral resolution of $75 \mathrm{~nm}$ is predicted with the advantage of a regular confocal detection. Finally, we show how using these techniques in combination with a two-color two-photon excitation could permit to further improve the resolution to $60 \mathrm{~nm}$.

PACS numbers:

Keywords: point spread function engineering, fluorescence microscopy, image formation 


\section{Introduction}

The resolution in optical microscopy is limited by the diffraction effect. One century ago, Ernst Abbe [1] introduced (for conventional microscopy) his resolution criteria as:

$$
\mathrm{R}_{\mathrm{Abbe}}=0.5 \lambda / \mathrm{NA}
$$

with $\lambda$ being the wavelength of observation and NA being the numerical aperture of the objective, defined as $\mathrm{NA}=\mathrm{n} \sin \alpha$. In this formula, $\mathrm{n}$ represents the index of refraction of the observation medium, and $\alpha$ is the maximum angle of collection of the objective.

For years, this limit was considered as ultimate. Marvin Minski made a first progress by inventing the confocal microscope [2], in which the specimen is not anymore uniformly illuminated, but using a focalized beam, and a detection pinhole cutting most of the out-of-focus light. For a NA=1.4 objective observing into a watery medium, an excitation at $\lambda_{\text {exc }}=400 \mathrm{~nm}$ and a detection at $\lambda_{\text {det }}=450 \mathrm{~nm}$, the theoretical confocal lateral resolution is $130 \mathrm{~nm}$.

Recently, the development of new techniques like structured illumination [3] and STED microscopy [4] has permitted to further improve the resolution. Structured illumination however requires taking several images of the same specimen to recompose a resolution-improved image, which may be a drawback for bleaching-sensitive dyes. Up to now, STED microscopy, while very promising, has been proven for a very small number of dies only. We propose a technique, which should deliver a sub-100 nm lateral resolution for readily available dyes.

In order to get the best resolution, the use of the largest numerical aperture objective is mandatory (See Eq. (1)). Two types of high NA objectives are currently used. Oil immersion objectives with $\mathrm{NA}=1.4$ are available, giving a theoretical Abbe resolution of $160 \mathrm{~nm}$ when observing at $\lambda_{\text {det }}=450 \mathrm{~nm}$. These objectives are however not well suited for 3-D observations, because the refractive index difference between the immersion oil $\left(\mathrm{n}_{\mathrm{oil}}=1.515\right)$ and the specimen (usually assimilated to water $\mathrm{n}_{\text {spec }}=1.33$ ) induces spherical aberration, which rapidly degrades the microscope performances. In order to limit spherical aberration, water immersion objectives with NA=1.2 have been developed. The 
resolution they offer is therefore slightly lower, but this resolution is kept constant for 3-D observations.

Throughout this work, we focus however our attention to objects, which can be considered as 2-D or pseudo 2-D, like for examples chromosomes in the metaphase state, deposited on a glass slide. Similarly, microtubules after extraction from a cell, or DNA fragments may even be considered as 1-D samples. These specimens under examination not being living, one can embed them in an appropriate medium with an index of refraction closely matching that of the immersion medium.

To this date, the objective with the highest available numerical aperture has been developed by Olympus for Total Internal Reflection Fluorescence microscopy [5]. This objective with NA=1.65 requires a special immersion oil with $\mathrm{n}_{\text {oil }}=1.78$ and special coverslips with $\mathrm{n}_{\text {glass }}=1.788$. We first propose to use this special objective in a confocal configuration, with a preparation of the pseudo 2-D specimens under consideration within the same oil, in order to avoid total internal reflection. We consider the Cascade Blue dye from Molecular Probes, which has an excitation maximum at $\lambda_{\text {exc }}=400 \mathrm{~nm}$ and emits near $\lambda_{\text {det }}=450 \mathrm{~nm}$.

When considering such high NA objectives, scalar theories may fail to correctly predict the shape of the Point Spread Function (PSF). To compute the illumination PSF $\mathrm{ill}_{\text {ill }}$, we therefore consider the vectorial theory of Török and Varga [6], modified by Haeberlé [7]. The detection PSF $_{\text {det }}$ is computed using the vectorial dipole model of Haeberlé et al. [8] similarly adapted to biological cases by Haeberlé [9], in order to take into account the objective manufacturer's specifications as in Ref. [10].

The total confocal PSF conf $_{\text {in }}$ given by the product of the illumination PSF ill $_{\text {and }}$ and detection PSF det $_{\text {. }}$ For the sake of simplicity, the illumination wave and the detected signal are supposed to be randomly polarized. In that case, the total confocal $\mathrm{PSF}_{\text {conf }}$ is circular symmetric.

Figure 1 shows the obtained result for the considered dye. The confocal lateral resolution is $103 \mathrm{~nm}$, which already represents a $25 \%$ improvement compared to a confocal microscope using a standard NA=1.4 objective imaging in a watery medium. Indeed, this implies a decrease of the 
effective numerical aperture (to 1.33 at best), and the theoretical lateral confocal resolution is $130 \mathrm{~nm}$.

The aim of this work is to present a technique using laterally shifted and interfering illumination beams in order to improve the resolution (Note that in Ref. [4] another approach leading to a resolution enhancement is presented, also using lateral beam displacement, but based on a different phenomenon than interferences). Narrowing the PSF is equivalent to better preserving in the OTF (the Fourier transform of the PSF) those frequencies that are under the cut-off frequency, which may be obtained by increasing the strength of the higher frequencies, or damping the continuous and low frequencies. This may also help for the restoration of the image by deconvolution.

\section{Modified illumination}

In order to further improve the lateral resolution, we first propose to use a modified illumination $\mathrm{PSF}_{\mathrm{ill}}$. The technique is to insert in the illumination arm of the confocal microscope a phase plate, which reverts the sign of the amplitude of the illumination wave in one-half of the entrance pupil. Such a phase plate was used in a different context, in order to create a beam with a one-direction valley of depletion, in order to test beams with various profiles for STED microscopy [11]. We propose to use this kind of phase plate in a regular confocal microscope, in order to diminish the size of the excitation $\mathrm{PSF}_{\mathrm{ill}}$, the detection $\mathrm{PSF}_{\mathrm{det}}$ remaining unmodified.

We consider focusing by a high numerical aperture lens of an illumination plane wave of wavevector $\mathrm{k}_{0}$, linearly polarized along the $\mathrm{x}$-axis. The electric field is determined at the point $\mathrm{P}(\mathrm{x}, \mathrm{y}, \mathrm{z})$ in the focal region. Throughout this work, we use spherical polar coordinates $\left(r, \theta_{p}, \varphi_{p}\right)$ with $r>0,0 \leq \theta_{p}<\pi$ and $0 \leq \varphi_{\mathrm{p}}<2 \pi$. For the sake of simplicity, Figure 2 depicts the considered geometry for focusing into a single medium, but we consider in the following focusing into a stratified medium composed of the immersion medium, coverslip, and specimen, of index $n_{1}, n_{2}$ and $n_{3}$, respectively. The immersion medium to coverslip and coverslip to specimen interfaces lay at coordinates $\mathrm{z}=-\mathrm{h}_{1}$ and $\mathrm{z}=-\mathrm{h}_{2}$, respectively [6-9]. 
In order to compute the corresponding PSF $_{\text {ill }}$ when using the non-rotationally symmetric phase plate, we use (neglecting some constant factors) the 2-D diffraction integrals proposed by Török and Varga [6]:

$$
\begin{aligned}
\mathbf{E}\left(r_{p}\right) & =\int_{0}^{\alpha} \int_{0}^{2 \pi} \boldsymbol{E} \exp \left(i r_{p} \kappa\right) \exp \left(i k_{0} \Psi_{i}\right) \\
& \times \exp \left(i k_{3} r_{p} \cos \theta_{p} \cos \theta_{n}\right) \sin \theta_{1} d \phi d \theta_{1}
\end{aligned}
$$

with:

$$
\boldsymbol{E}=A\left(\theta_{1}\right) B(\theta, \phi)\left[\begin{array}{c}
T_{p} \cos \theta_{3} \cos ^{2} \phi+T_{s} \sin ^{2} \phi \\
\left(T_{p} \cos \theta_{3}-T_{s}\right) \cos \phi \sin \phi \\
-T_{p} \sin \theta_{3} \cos \phi
\end{array}\right]
$$

The function $\operatorname{A}(\theta)$ is the apodization function, which for an aberration-free system obeying the sine condition is given by $\mathrm{A}(\theta)=\cos ^{1 / 2} \theta$.

The function $\mathrm{B}(\theta, \phi)$ describes the influence of phase plates used to modify the incident beam. We have introduced [6]:

$$
\begin{gathered}
\Psi_{i}=h_{2} n_{3} \cos \theta_{3}-h_{1} n_{1} \cos \theta_{1} \\
\kappa=k_{0} \sin \theta_{1} \sin \theta_{p} \cos \left(\phi-\phi_{p}\right)
\end{gathered}
$$

The transmission coefficient for a three-layer medium is given by:

$$
T_{s, p}=\frac{t_{12 s, p} t_{23 s, p} \exp (i \beta)}{1+r_{12 s, p} r_{23 s, p} \exp (2 i \beta)}
$$

with $\beta=k_{2}\left|h_{2}-h_{1}\right| \cos \theta_{2}$ and the Fresnel coefficients for transmission and reflection being given by:

$$
\begin{aligned}
t_{n n+1, s} & =\frac{2 n_{n} \cos \theta_{n}}{n_{n} \cos \theta_{n}+n_{n+1} \cos \theta_{n+1}} \\
t_{n n+1, p} & =\frac{2 n_{n} \cos \theta_{n}}{n_{n+1} \cos \theta_{n}+n_{n} \cos \theta_{n+1}} \\
r_{n n+1, s} & =\frac{n_{n} \cos \theta_{n}-n_{n+1} \cos \theta_{n+1}}{n_{n} \cos \theta_{n}+n_{n+1} \cos \theta_{n+1}} \\
r_{n n+1, p} & =\frac{n_{n+1} \cos \theta_{n}-n_{n} \cos \theta_{n+1}}{n_{n+1} \cos \theta_{n}+n_{n} \cos \theta_{n+1}}
\end{aligned}
$$

For fluorescence microscopy, we consider the intensity Point Spread Function at illumination: 


$$
\operatorname{PSF}_{i l l}(x, y, z)=|\mathbf{E}|^{2}=\left|\mathbf{E}_{x}+\mathbf{E}_{y}+\mathbf{E}_{z}\right|^{2}
$$

Corrective terms to take into account the objective's specifications, which take into account the coverslip characteristics, are introduced in Eqs. (4,5) as described in Ref. [7] for 1-D diffraction integrals. The phase plate we consider introduces a phase shift of $\pi$ between the two halves of the incident beam (as described by Fig. 3), so that:

$$
\mathrm{B}(\theta, \phi)=\operatorname{sign}(\phi-\pi)
$$

Figures 4(a) and 4(b) display the illumination $\mathrm{PSF}_{\text {ill }}$ obtained with this phase plate (Figure 4(c) and 4(d) show for comparison the regular PSF from an x-polarized excitation beam). It exhibits two lobes, with a valley oriented along the $\mathrm{x}$-axis, the peaks of maximum intensity being along the $y$-axis. Note that the correct orientation of the polarisation of the incident beam along the x-axis is critical to obtain such a Point Spread Function [11]. The Full Width Half Maximum (FWHM) of these lobes is only $105 \mathrm{~nm}$, much narrower than a standard $\mathrm{PSF}_{\mathrm{ill}}$ from the same objective with an unpolarized beam, which has a FWHM of $135 \mathrm{~nm}$.

Figure 5(a) shows the profile of this two-lobe PSF $_{\text {ill }}$ along the y-axis. Because the two lobes are well separated, it is possible to isolate one of them by confocalizing with a shifted pinhole (Figure 5(b) shows the corresponding detection $\mathrm{PSF}_{\mathrm{det}}$ computed using the simplified dipole radiation detection model as described in Ref. [9]). In the considered configuration, the pinhole is shifted $90 \mathrm{~nm}$ to the left, in order to coincide with the left maximum of the excitation PSF $_{\text {ill }}$. One obtains a final confocal $\mathrm{PSF}_{\text {conf }}$ with a FWHM of $90 \mathrm{~nm}$ (Figure 5(c)), showing that the proposed phase filtering permits a $12.5 \%$ improvement in the lateral resolution.

Note that for a more standard objective with $\mathrm{NA}=1.4$, the predicted confocal resolution with such a set-up (halve phase plate and special preparation of the sample) is still only $105 \mathrm{~nm}$. This result already represents a $25 \%$ improvement compared to a regular confocal microscope using the same objective and imaging into a watery medium. This shows that this simple technique may give a substantial gain in resolution also for more widely used objectives, and should attract the attention of 
users having interest in the observation of very shallow specimens.

The method described here is a peculiar case of pupil function engineering, used to modify the intensity distribution in the focal plane of a lens, for example to increase the spatial resolution, laterally or longitudinally, or in 3-D [12-18]. The difference is that instead of trying to optimize the central peak, interferences are used to produce two slightly smaller peaks.

\section{Laterally interfering excitation beams}

We have seen that a substantial improvement in lateral resolution is possible by using a phase plate creating destructive interferences at the position of the focal point. However, a careful examination of Figures 4(b) and 4(d) shows that the longitudinal resolution is degraded. It may have no practical consequences if one effectively studies ultra flat specimens, but indicates that the process is not optimal. This is easily explained when considering the Babinet principle. The interference process creates a dark fringe at the position of the regular focal point, which reduces the lateral extension of the two remaining spots, but the two interfering beams actually fill each only one-half of the backfocal plane of the objective. As a consequence, each half-beam produces a larger spot than for a beam filling the entire back-focal plane. Both interfere destructively along the y-axis, resulting in a PSF ill $_{\text {in }}$ made out of two smaller spots. The interference process occurs along the optical axis only (Fig. 4(b)) but not in the nearby, which explains that the resulting spots have a longer z-elongation.

In order to take benefit of the smallest possible focal spot, one must then use two or more focalized beams, which fill each the entire back-focal plane of the objective and are slightly shifted in the focal plane as well as properly dephased in order to benefit from a possible interference process. Focusing by a high numerical aperture objective induces partial depolarization at the focal spot. In order to properly compute the resulting point spread function one must therefore use a full vectorial theory. We use the model described in Ref. [7]. The intensity illumination $\mathrm{PSF}_{\mathrm{ill}}$ at point $\mathrm{P}(\mathrm{x}, \mathrm{y}, \mathrm{z})$ in the specimen (last layer of a three components stratified structure) can be computed with Eq. (7), the three 
components of the electromagnetic field being given by (with $\phi_{\mathrm{p}}$ in spherical polar coordinates):

$$
E_{x}=-i\left(I_{0 i l l}+I_{2 i l l} \cos 2 \phi_{p}\right), E_{y}=-i\left(I_{2 i l l} \sin 2 \phi_{p}\right), E_{z}=-2 I_{1 i l l} \cos \phi_{p}
$$

For a detailed description of how to compute the three diffraction integrals $I_{0}, I_{1}$ and $I_{2}$, the interested reader is referred to Refs. [6-10]. We here just recall that $I_{0}, I_{1}$ and $I_{2}$ are radialy symmetric with respect to the optical axis, and in the absence of aberrations, symmetric with respect to the focal plane. The use of a linearly polarized incident beam however implies an asymmetric point spread function, because of the $\phi_{\mathrm{p}}$ dependence in Eq. (10), and $\mathrm{PSF}_{\mathrm{ill}}$ is slightly narrower along the y-axis than along the $\mathrm{x}$-axis (for a x-polarized beam) [19].

Figure 6 describes the considered configuration, with two x-polarized incident beams giving rise to

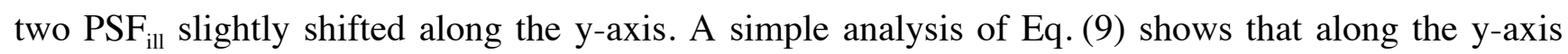
$\left(\phi_{\mathrm{p}}=90^{\circ}\right.$ for first beam, $\phi_{\mathrm{p}}=270^{\circ}$ for second beam), $\mathrm{E}_{\mathrm{y}}$ and $\mathrm{E}_{\mathrm{z}}$ are null, and both $\mathrm{E}_{\mathrm{x}}$ components may interfere at point $\mathrm{M}$ for beams shifted along the $\mathrm{y}$-axis. For $\mathrm{x}$-axis shifted beams however, $\left(\phi_{\mathrm{p}}=0^{\circ}\right.$ for first beam, $\phi_{\mathrm{p}}=180^{\circ}$ for second beam), the $\mathrm{E}_{\mathrm{y}}$ components are null, but having the $\mathrm{x}$-components to destructively interfere implies that the z-components add at point M (See Eq. (9)). As a consequence, it is not possible to have a fully destructive interference between both beams in this configuration.

The best results are however obtained not with a two spots interference scheme, but with a three spots interference process, the central spot being narrowed by two lateral neighboring spots. Figure 7(a) show the (x-y) extension of the illumination spot with same parameters as for Fig. 5. Figure 7(b) displays the profile of the excitation PSF $_{\text {ill }}$ obtained with this method, the detection PSF $_{\text {det }}$ computed at

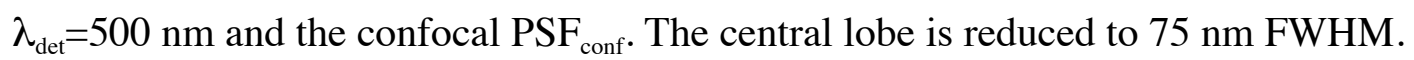

Note that in this configuration, the confocalization process does not improve (or very marginally) the resolution. On the contrary, in a classical confocal microscope, the illumination and detection PSFs are of similar spatial extension (the detection $\mathrm{PSF}_{\mathrm{det}}$ being wider because of the Stokes shift), so that multiplying both PSFs leads to a lateral resolution increase of about $40 \%$ with an infinitely small 
pinhole. However, in order to efficiently collect fluorescence photons, one must use in practice a pinhole of size similar to the Airy disk, and as a consequence, the gain in lateral resolution is much smaller, the main interest of the confocal setup being its better optical sectioning capabilities, compared to a wide field microscope.

In the proposed configuration, the resolution is in fact effectively determined by the three-beam illumination process. This as the important practical consequence that opening the pinhole in order to get more collected photons will not noticeably degrade the resolution, contrary to the usual confocal microscope.

Confocalizing is however mandatory to suppress the side-lobes. When opening the pinhole, the height of the remaining side-lobes will increase, which is unfavorable, as it may induce the apparition of ghost images surrounding the main image. The situation in that case is very similar to 4Pi-confocal microscopy [20], for which fast linear mathematical operation have been developed to effectively remove this ghost images [21]. Similar processing may therefore be used for this new kind of confocal microscopy with compound excitation.

Other techniques do permit a significant gain in resolution, as for example using an annular aperture [12], which would be simpler to implement than the method we proposed. With an aperture stop half the size of the NA=1.65 aperture, the predicted resolution is $85 \mathrm{~nm}$, versus $75 \mathrm{~nm}$ with the three-beam illumination scheme, for excitation at $400 \mathrm{~nm}$ and detection at $500 \mathrm{~nm}$. It would possible to go down to $75 \mathrm{~nm}$ with an aperture stop of $90 \%$ of the objective aperture. This configuration would however present two major drawbacks compared to ours: first, this configuration blocks $81 \%$ of the incident energy. Secondly, out of the $19 \%$ transmitted energy, most is dispersed in numerous high intensity diffraction rings, so that only a very small fraction of the incident energy is really concentrated in the central peak. Our three-beam setup would require dividing the incident beam into three, so that still roughly $30 \%$ of the energy remains in the central peak. Furthermore, using a central aperture block cannot permit to split the focal spots into two smaller spots (as when using the halves 
phase plate, or with a two-beam interference scheme), a technique which one may take benefit from to even further improve the resolution as explained in the next section.

\section{Two-Color Two-Photon excitation}

We now consider the case of two-color two-photon (2C2P) excitation, which has also been recently proposed for confocal [22-24] and 4Pi microscopy [25], and has some advantages over one-color twophoton excitation when using apodized illumination [26] or when observing through a scattering medium [27]. In the two-color two-photon excitation process, two photons with different wavelength contribute to excite the fluorophore. The one-color two-photon excitation process used in two-photon microscopy may therefore be considered as a special case of the more general two-color two-photon excitation process, in which both photons have the same wavelength. Very few dyes have for the moment proven to be excited using a two-color two-photon scheme. However, some of them belong to the Coumarin family [28], already used in fluorescence microscopy, which opens the possibility of having biologically compatible two-color two-photon dyes.

Figure 8(a) shows $\mathrm{PSF}_{\mathrm{ill}}$ at at $400 \mathrm{~nm}$ using a x-polarized beam passing through the halves phase plate and an annular aperture with transmittance 0 for $\theta \leq 0.9 \alpha$. The use of an annular aperture permits to get a double lobe PSF with slightly compressed lobes along the y-axis. The price to be paid is that higher order interference rings are of higher intensity than with a regular full aperture. We will show that is not a problem, thanks to the $2 \mathrm{C} 2 \mathrm{P}$ excitation process.

Figure 8(b) shows $\mathrm{PSF}_{\mathrm{ill} 800}$ at $800 \mathrm{~nm}$ without phase plate. The excitation beam is also x-polarized in order benefit from the slight gain in resolution again the y-axis when using polarized excitation [19], and the same annular aperture with transmittance 0 for $\theta \leq 0.9 \alpha$ is used. Again, higher rings do appear.

Because of the two-photon process, the total $\mathrm{PSF}_{\mathrm{ill2} 2 \mathrm{C} P}$ is given by $\mathrm{PSF}_{\mathrm{ill180}} \times \mathrm{PSF}_{\mathrm{ill400}}$. However, because $\mathrm{PSF}_{\mathrm{ill1800}}$ exhibits a central peak, while $\mathrm{PSF}_{\mathrm{ill400}}$ has two offset lobes, the resulting $\mathrm{PSF}_{\mathrm{ill2C} 2 \mathrm{P}}$ is also characterized by two out-of-axis peaks. This is illustrated by the curve in solid line on Figure 8(c). 
The product of the two PSFs, having higher interference rings, but located at different positions, results in a final $\mathrm{PSF}_{\mathrm{ill2} 2 \mathrm{C}}$ with no noticeable rings.

The configuration we describe is in fact a variant of the two-photon excitation with laterally offset beams proposed by Stefan Hell [29]. However, for one-color two-photon excitation with two laterally offset beams, three different contributions define the final $\mathrm{PSF}_{\mathrm{ill}}$. The central, narrower peak is defined by the product of the two offset PSFs, as described in Ref. [29], but both lateral beams may also individually induce a one-color two-photon excitation. In 2C2P excitation, because each beam alone cannot induce a two-photon excitation, only one process finally contributes to the excitation.

Again, the two separated lobes may be confocalized. For detection at $350 \mathrm{~nm}$ [28], shifted by $65 \mathrm{~nm}$ to coincide with the maximum of the $2 \mathrm{C} 2 \mathrm{P}$ curve (illustrated on Figure 8(c) by the dashed line), the final resolution is $60 \mathrm{~nm}$ at FWHM. Figure 8(d) shows the total PSF $_{\text {conf }}$ for the left pinhole (solid line) and for the right pinhole (dashed line).

\section{Discussion}

The three-beam illumination method produces two very high side-lobes in the illumination PSF $\mathrm{ill}_{\text {, }}$ which indeed are suppressed after the product with the collection PSF $_{\text {det }}$. However these illumination side-lobes can be very detrimental, because, during the scanning process, they can bleach the fluorescent dye before the signal normally occurring from the central peak is actually acquired. This obviously constitutes a limitation for the adoption of this technique, which should be used only with bleaching resistant dyes.

This remark also holds when using a dual lobe illumination PSF $_{\text {ill }}$ (halve plate, dual beam with or without two-color two-photon excitation) for which another drawback of the proposed confocalization technique is that using only one axially offset pinhole represents in fact a waste of signal, while obviously the same information could be obtained from the left- or the right lobe, as they are symmetric (note that for the three-beam illumination, only the central peak permits the best 
resolution). However, because the lobes are well separated in the (x-y) imaging plane, it should be possible to use two pinholes properly centered at the positions of both maxima, with two independent detectors to simultaneously acquire during the scanning process two independent images of the specimen. To do so, one should modify the detection channel (compared to a confocal microscope using regular illumination) with an intermediary magnifying optics, so that the lobes visible on the graphs of the split PSFs (Figs. 5 and 8) have a spatial separation in the image plane slightly larger than the diameter of the pinhole, which is typically a few tens of micrometers. Another solution could be to use optics fibers as pinholes to collect the light: the small diameter of their core would not imply the use of large magnifications, and they offer a great flexibility of design to carry the photons to the detectors. The photon detection efficiency constitutes a possible important limitation in practice, because without dual detection, the halves phase plate implementation would have similar resolution as other pupil plane mask design [12-18], but only half the detection efficiency. The $2 \mathrm{C} 2 \mathrm{P}$ scheme keeps the advantage of its very high resolution.

When the two peaks are optically separated, both obtained images contain each the signal emitted by the same object point illuminated, during the scanning process, in one image with the left lobe of the two-lobe $\mathrm{PSF}_{\mathrm{ill}}$, and in the second image with the right lobe, and detected with the left- and the right confocal detector, respectively. These two images can then be recombined by a simple shiftingadding method, which would have for effect to simultaneously improve the signal to noise ratio and to decrease the height of the remaining side-lobe of the confocal $\operatorname{PSF}_{\text {conf }}$ (see Figure 4(c)) relatively to the main peak.

Obviously, the simplest technique is to use the halve phase plate, but the three-beam method cumulates both advantages that it offers a better lateral resolution while keeping a centered detection pinhole, which is probably easier to setup and maintain. The most difficult point is to properly shift and dephase the lateral beams so as to induce the interference process. One can think at two ways to obtain this shift. 
The first technique is to inject tilted beams into the objective's back focal plane. However, because of the high magnification $(63 \mathrm{x}, 100 \mathrm{x})$ of high NA objective, and because of the very small shifts needed (a few tens of nanometers), this technique would imply minute tilting angles, probably difficult to finely adjust and keep stable.

A second technique is to impose to the field incoming onto the objective's back focal plane a phase gradient, which turns out as a lateral translation in the focal plane. This may be obtained by injecting three beams into the back focal plane of the objective, all parallel to the optical axis, with one regular beam (which will give rise to the central spot), and the two other beams passing through properly designed phase plate add the correct phase gradient. A steady state alternative to phase plate could be the use of Wollaston prisms, usually used in differential interference contrast (DIC) microscopy, as Wollaston prisms actually act as adjustable phase gradient elements [30,31].

Another potential issue is the possible presence of aberrations because of a mismatch between the special oil with a very high index of refraction of 1.788 , and the observed specimen, when using $\mathrm{NA}=1.65$ objectives in order to get the highest resolution. Even small aberrations may have a significant negative impact on the resolution, because of the very large angle of incidence of the marginal rays of the excitation beam. We have therefore studied the influence of slight changes of the refraction index of the mounting medium for the observed specimen. We have considered a specimen embedded in a fixing solution with index of refraction 1.70 (instead of the nominal $\mathrm{n}_{\mathrm{oil}}=1.788$ ) at a depth of $5 \mu \mathrm{m}$ below the cover glass, as we now that even slight mismatch between the immersion medium and the specimen induces a noticeable degradation of the illumination- as well as of the detection PSFs, and also has a strong impact on deconvolved images [32]. All other parameters are assumed to be nominal. Computations of the illumination and detection PSFs show in this case that the longitudinal resolution is mostly affected, both exhibiting high interferences fringes, and being strongly asymmetric with respect to the focal plane. This effect is well known [6-10]. If one considers very flat specimens only, one is however more concerned with the lateral resolution. In such 
conditions, for a confocal set-up with $\lambda_{\text {exc }}=400 \mathrm{~nm}$ and $\lambda_{\text {det }}=450 \mathrm{~nm}$, the lateral resolution is $130 \mathrm{~nm}$, compared to $103 \mathrm{~nm}$ with nominal conditions (Fig. 1), showing that even the lateral resolution is sensitive to small errors in the sample preparation process.

Furthermore, the distributions of the $\mathrm{x}-, \mathrm{y}-$, and $\mathrm{z}$-polarized components of the illumination $\mathrm{PSF}_{\mathrm{ill}}$ are differently affected by the presence of spherical aberrations. As a consequence, obtaining lateral interferences as proposed in Part 3 requires different beam lateral shifts than with nominal parameters (Fig. 6). This constitutes of course a major obstacle for this technique. Tightly mastering the preparation of the sample, which must closely match the index of refraction of the special immersion oil, therefore appears to be mandatory if one wants to use the special N.A.=1.65 objectives combined with lateral interferences.

In order to avoid this aberration problems, especially for living specimens and for 3-D observations, a solution is to use water immersion objectives with $\mathrm{NA}=1.2$. Note that even for such objectives with a much lower N.A., the three-beam excitation scheme, combined with a confocal detection would still provide a (theoretical) lateral resolution of $104 \mathrm{~nm}$ at $\lambda_{\text {exc }}=400 \mathrm{~nm}$ and $\lambda_{\mathrm{det}}=500 \mathrm{~nm}\left(\mathrm{R}_{\mathrm{Abbe}}=209 \mathrm{~nm}\right)$.

\section{Conclusion and perspectives}

In summary, we have shown that using the highest numerical aperture objectives now available, combined with an appropriate preparation of the specimen to match the index of refraction of the objective immersion oil, and using a simple halves plate apodization technique opens the way to sub$100 \mathrm{~nm}$ resolution for 2-D specimens in far-field fluorescence microscopy, with a predicted lateral resolution of $90 \mathrm{~nm}$.

The halves phase plate technique is however not optimal, and we have proposed an alternate method, based on laterally interfering beams, which permits to improve the lateral resolution in a confocal microscope to $75 \mathrm{~nm}$ with readily available dyes. 
When combined with a two-color two-photon excitation scheme, the resolution may even be further improved to about $60 \mathrm{~nm}$. At present time, there are however very few dyes, which are compatible with this technique.

The three proposed methods permit a direct lateral gain along one direction only. So, strictly speaking, the method is directly useful for 1-D specimens only, like microtubules, or DNA fragments. A gain in resolution in 2-D can however readily be obtained by acquiring two or more successive images with improved resolution along several directions, which are simply recombined, as already done in 3-D for the Multiple Imaging Axis Microscope [33]. Similarly, one can also take benefit of the several 2-D images, which are different versions of the same sample, perturbed by different blurs to improve the kernel estimation and the deconvolution process [34].

In order to obtain a direct 2-D improvement of the lateral resolution without having to recombine several images, one may think at studying beams with radial or azimuthal polarization, or more generally Gauss-Laguerre beams, as they offer a greater flexibility to modify the PSF ill $_{\text {when }}$ using high NA objectives [35].

A major drawback is that none of the three methods we have proposed does permit to improve the axial resolution. This is because we have considered lateral interferences only. Note however that these methods are fully compatible with the 4Pi microscope of type A, in which counter propagating beams, passing through two opposing lenses, interfere longitudinally. This scheme permits to greatly enhance the longitudinal resolution, but the lateral resolution remains that of a regular confocal microscope $[20,21]$. In this configuration, water immersion objectives with a lower NA of 1.2 must be used in order to study living specimens, but anyway, a longitudinal resolution of about $110 \mathrm{~nm}$ has already been proven, also with a commercially available instrument [36]. Combining both techniques of lateral and longitudinal interferences represents a thorough challenge, but is probably easier than using a multiple objective approach as proposed in Ref. [37,38]. It may open the way to a quasiisotropic resolution near $100 \mathrm{~nm}$ in the three dimensions and into a biological sample, a result which 
has up to know been directly obtained (without the help of further image post-processing like deconvolution) only with STED microscopy [39].

\section{Aknowledgements:}

The authors gratefully acknowledge Peter Török and Peter Munro for enlightening discussions about techniques used for optical beam shaping and PSF engineering. The authors also would like to acknowledge two anonymous referees, whose comments and suggestions helped to improve the quality of the paper. 


\section{References}

1. Abbe E., Arch. Mikrosk. Anat. 9, p. 413 (1873)

2. D. Minsky, Scanning 10, p. 128 (1988)

3. M.G.L. Gustafsson, J. Microsc. 198, p. 82 (2000)

4. T. A. Klar and S.W. Hell, Opt. Lett. 24, p. 954 (1999)

5. see: http://www.olympusmicro.com/primer/techniques/fluorescence/tirf/olympusaptirf.html

6. P. Török and P. Varga, Appl. Opt. 36, p. 2305 (1997)

7. O. Haeberlé, Opt. Comm. 216, p. 55 (2003)

8. O. Haeberlé et al., Opt. Exp. 11, p. 2964 (2003)

9. O. Haeberlé, Opt. Comm. 235, p. 1 (2004)

10. S.F. Gibson and F. Lanni, J. Opt. Soc. Am. A 8, p. 1601 (1991)

11. T.A. Klar, E. Engel and S.W. Hell, Phys. Rev. E 64, 066613 (2001)

12. C.J.R. Sheppard and T. Wilson, Appl. Opt. 18, p. 3764 (1979)

13. Z. Hegedus and V. Safaris, J. Opt. Soc. Am. A 3, p. 1892 (1986)

14. C.J.R. Sheppard, Optik 99, p. 32 (1995)

15. M.A.A. Neil et al., Opt. Lett. 25, p. 245 (2000)

16. M. Martinez-Corral et al., Opt. Lett. 26, p. 1861 (2001)

17. M. Martinez-Corral, C. Ibàñez-López, and G. Saavedra, Opt. Exp. 11,p. 1740 (2003)

18. M. Yun, L. Liu, J. Sun, and D. Liu, J. Opt. Soc. Am. A 22, p. 272 (2005)

19. B. Richards and E. Wolf, Proc. R. Soc. London Ser. A 253, p. 358 (1959)

20. S.W. Hell and E.H.K. Stelzer, J. Opt. Soc. Am. A 9, p. 2159 (1992)

21. S.W. Hell, "Increasing the resolution of far-field fluorescence light microscopy by point-spreadfunction engineering" in Topics in Fluorescence Spectroscopy; Vol. 5: Nonlinear and Two-Photon- 
Induced Fluorescence, J. Lakowicz ed., Plenum Press, New York (1997)

22. Ma. O. Cambaliza and C. Saloma, Opt. Comm. 184, p. 25 (2000)

23. M. Lym and C. Saloma, Opt. Comm. 207, p. 121 (2002)

24. F. Xiao, G. Wang and Z. Xu, Opt. Comm. 228, p. 225 (2003)

25. J. Chen and K. Midorikawa, Opt. Lett. 29, p. 1354 (2004)

26. M.T. Caballero et al., Opt. Comm. 246, p. 313 (2005)

27. C. M. Blanca and C. Saloma, Appl. Opt. 40, p. 2722 (2001)

28. J. Palero, W. Garcia and C. Saloma, Opt. Comm. 211, p. 65 (2002)

29. S.W. Hell, Opt. Comm. 106, p.19 (1994)

30. P.R.T. Munro and P. Török, "A high numerical aperture, vectorial theory of confocal, scanning differential interference contrast microscope", presented at Focus on Microscopy 2005, Jena (Germany), March 20-23, 2005. Technical Digest of Focus in Microscopy 2005, p. 29 (2005)

31. P.R.T. Munro and P. Török,, Private Communication

32. O. Haeberlé et al., Opt. Comm. 196,p. 109 (2001)

33. J. Swoger, J. Huisken and E.H.K. Stelzer, Opt. Lett. 28, p. 1654 (2003)

34. F.Goudail, O.Ruch and P. Réfrégier, Appl. Opt., 39, p. 6602 (2000)

35. P. Török and P. R. T. Munro, Opt. Exp. 12,p. 3605 (2004) http://www.opticsexpress.org/abstract.cfm?URI=OPEX-12-15-3605

36. H. Gugel, et al., Biophys. J. 87, p. 4146 (2004).

37. O. Haeberlé et al., Opt. Lett. 26, p. 1684 (2001)

38. O. Haeberlé et al., Compt. Rend. Acad. Sciences IV (Paris) 2, p. 1509 (2001)

39. T. A. Klar et al., Proc. Natl. Acad. Sci. USA 97, p. 8206 (2000) 


\section{Figure Captions}

Fig. 1: Confocal PSF when using a N.A.=1.65 objective with a specially prepared 2-D specimen, and when using a N.A.=1.4 objective observing into water, with excitation at $400 \mathrm{~nm}$ and detection at $450 \mathrm{~nm}$.

Fig. 2: Diagram showing light focused by an apodized lens (for the sake of simplicity, a single medium is depicted).

Fig. 3: Halves phase plate used to modify the illumination $\mathrm{PSF}_{\mathrm{ill}}$ in a confocal set-up. It splits the incoming beam in two parts, which are dephased by $\pi$.

Fig. 4: Two-lobe illumination PSF $_{\text {ill }}$ at $400 \mathrm{~nm}$ with the halve phase plate of Fig. 3 and a N.A.=1.65 objective. (a): (x-y) and (b): (y-z) profiles (The (x-y) arrows are $200 \mathrm{~nm}$ long. Note that the scale along Z-axis is doubled). (c) and (d) show for comparison the regular illumination PSF with no phase plate.

Fig. 5: Profiles along the y-axis of (a) two-lobe PSF $_{\text {ill }}$ of Fig. (4) with excitation at $400 \mathrm{~nm}$, (b) shifted detection at $450 \mathrm{~nm}$, and (c) resulting confocal $\mathrm{PSF}_{\text {conf }}$. The resolution at FWHM is $90 \mathrm{~nm}$.

Fig. 6: Scheme to construct interferences between two point spread functions, resulting from xpolarized beams, and slightly shifted along the y-axis.

Fig. 7: (a): $(x-y)$ three-beam PSF $_{\text {ill }}$ resulting from the interaction of a central $x$-polarized PSF $_{\text {ill }}$ interfering with two lateral x-polarized $\mathrm{PSF}_{\text {ill }}$ shifted along the y-axis (The (x-y) arrows are $200 \mathrm{~nm}$ long). (b) y-axis profiles of the illumination three-beam PSF $_{\text {ill }}$ (solid line), the detection PSF $_{\text {det }}$ (dashed line), and the confocal PSF $_{\text {conf }}$ (dotted line). The resolution at FWHM is $75 \mathrm{~nm}$. 
Fig. 8: Profiles along the y-axis of (a) annular excitation at $400 \mathrm{~nm}$ with phase plate, (b) annular excitation at $800 \mathrm{~nm}$, (c) 2C2P excitation (solid line), and detection at $350 \mathrm{~nm}$ (dashed line), and (d) final 2C2P confocal $\mathrm{PSF}_{\text {conf }}$ for a left- (solid line) or a right-shifted detection (dashed line). The resolution at FWHM is $60 \mathrm{~nm}$. 


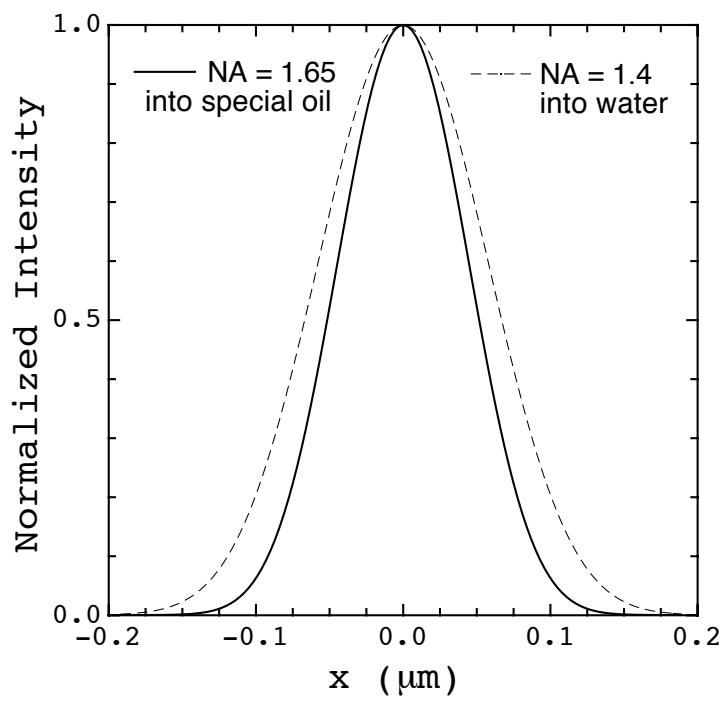

O. Haeberlé, "Improving the lateral resolution ......" Figure 1 


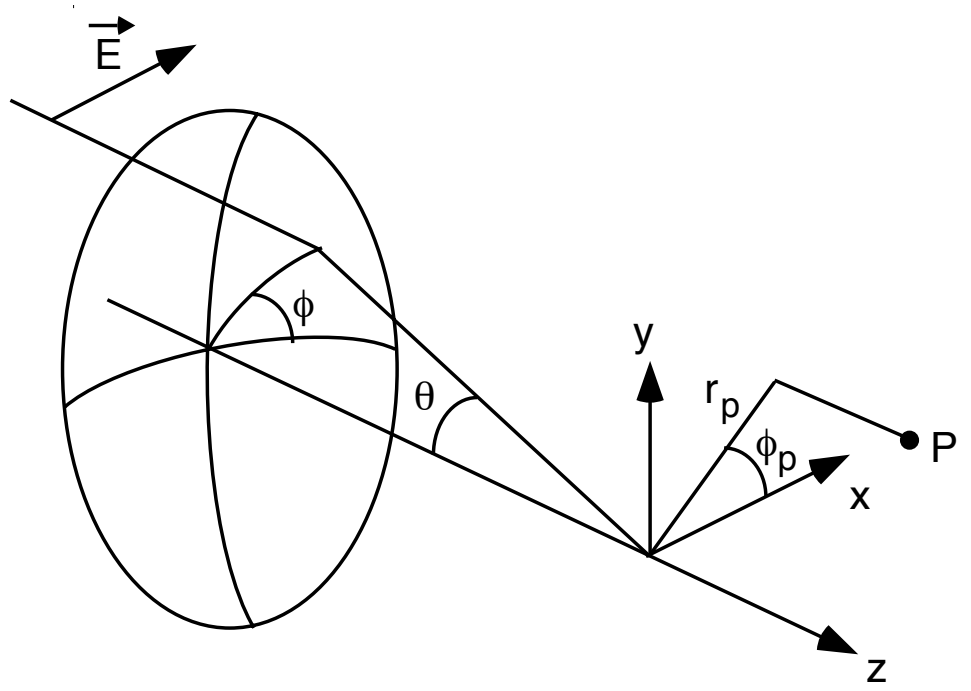

O. Haeberlé, "Improving the lateral resolution ......" Figure 2 


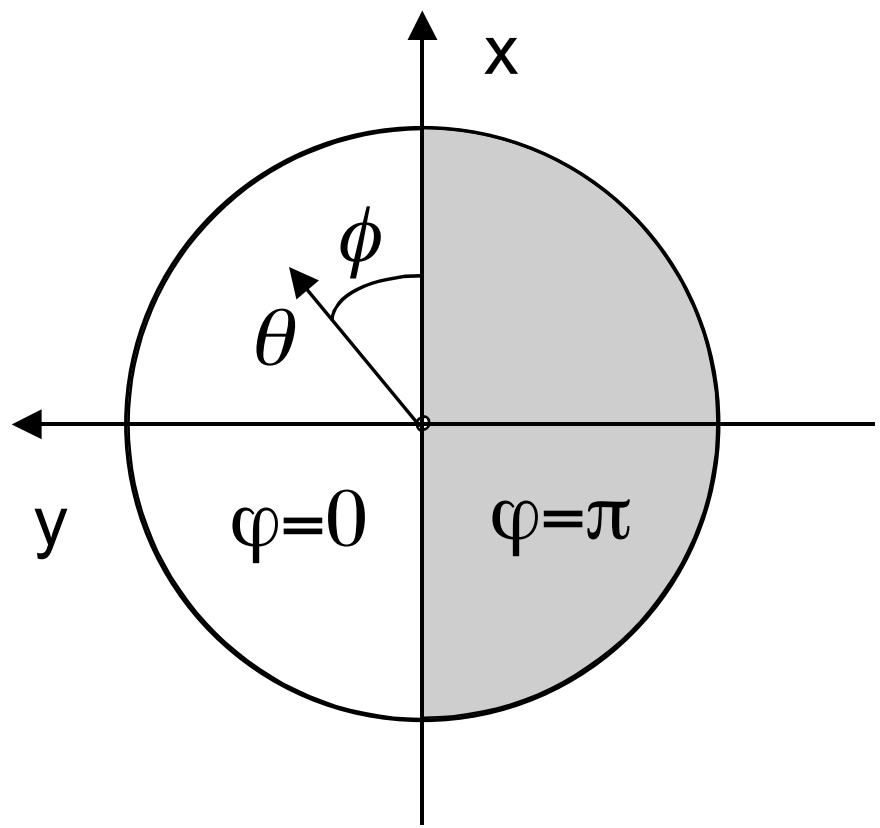

O. Haeberlé, "Improving the lateral resolution ......" Figure 3 


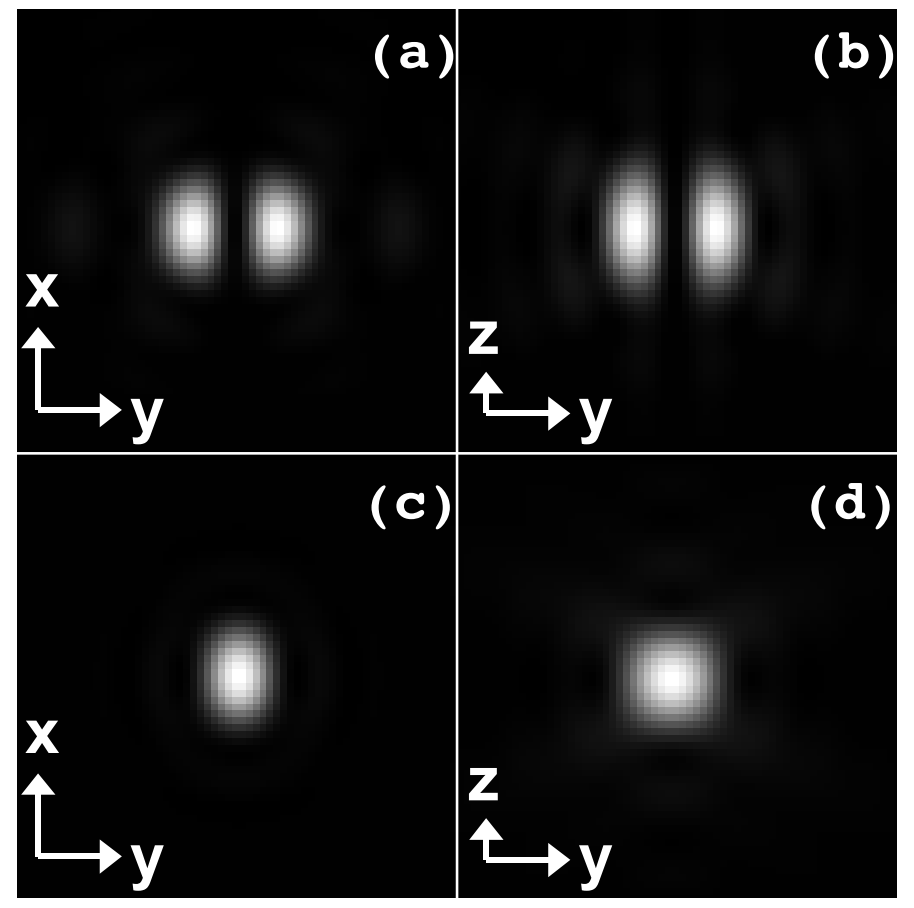

O. Haeberlé, "Improving the lateral resolution ......" Figure 4 

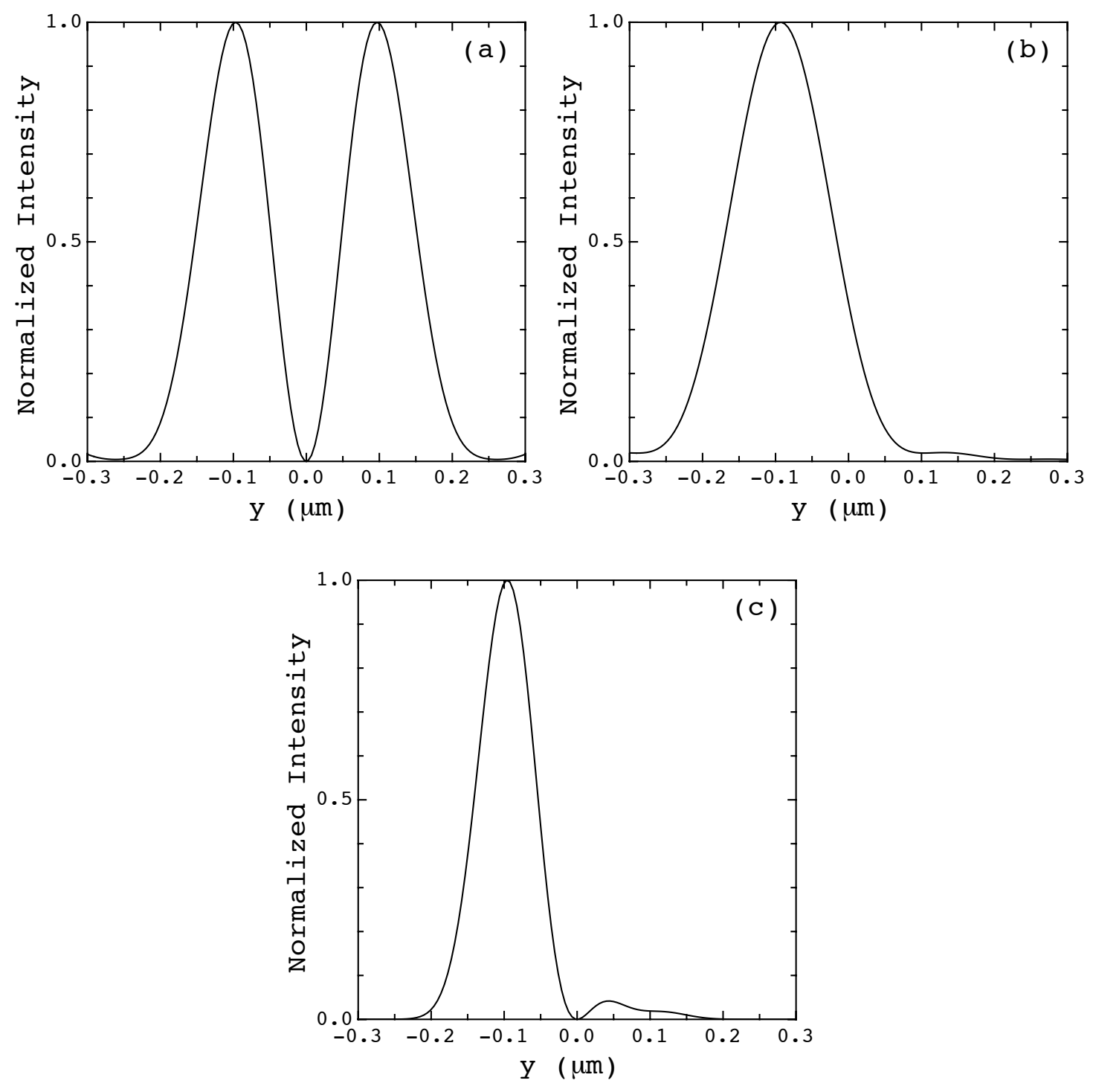

O. Haeberlé, "Improving the lateral resolution ......" Figure 5 


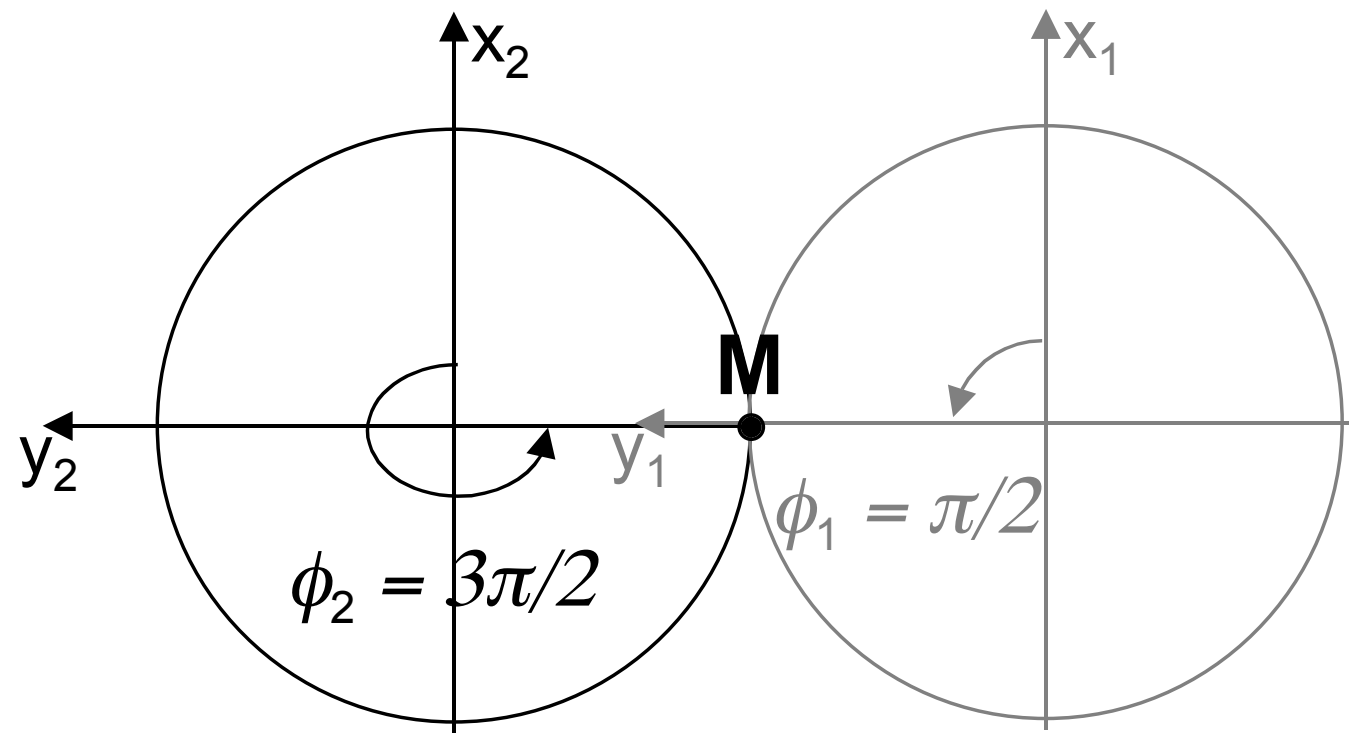

O. Haeberlé, "Improving the lateral resolution ......" Figure 6 

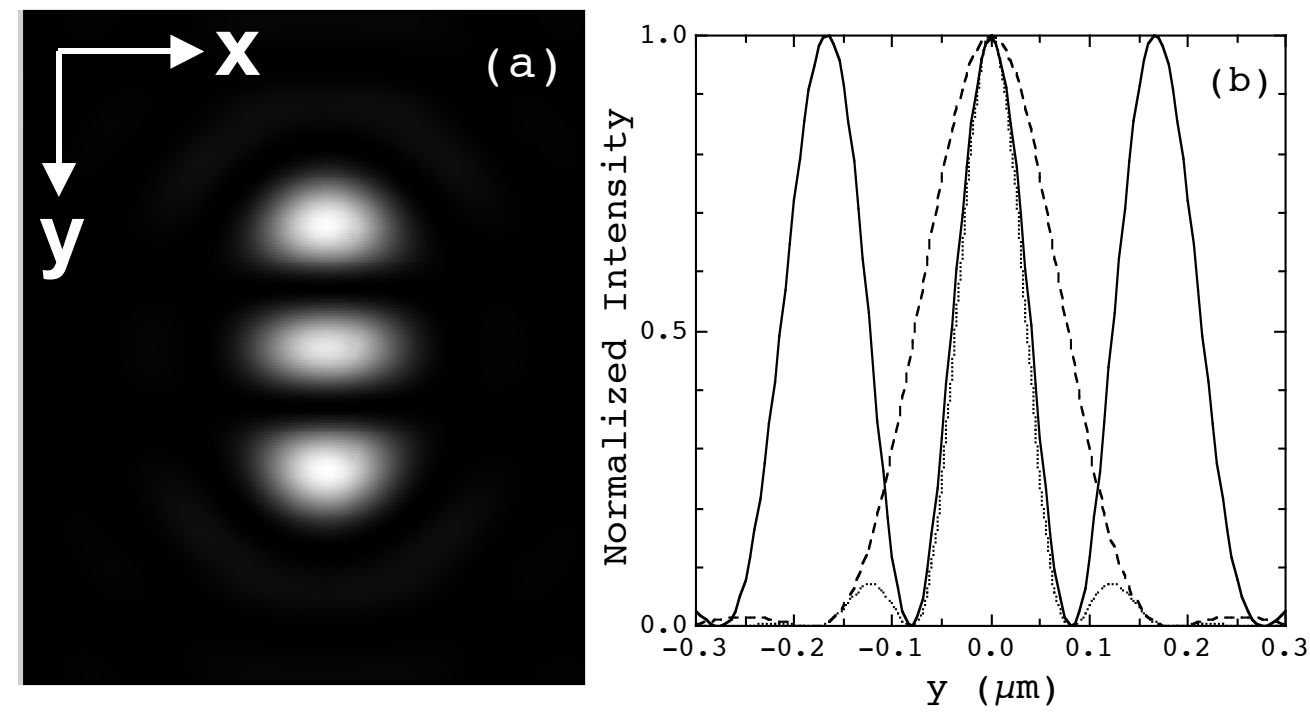

O. Haeberlé, "Improving the lateral resolution ." Figure 7 

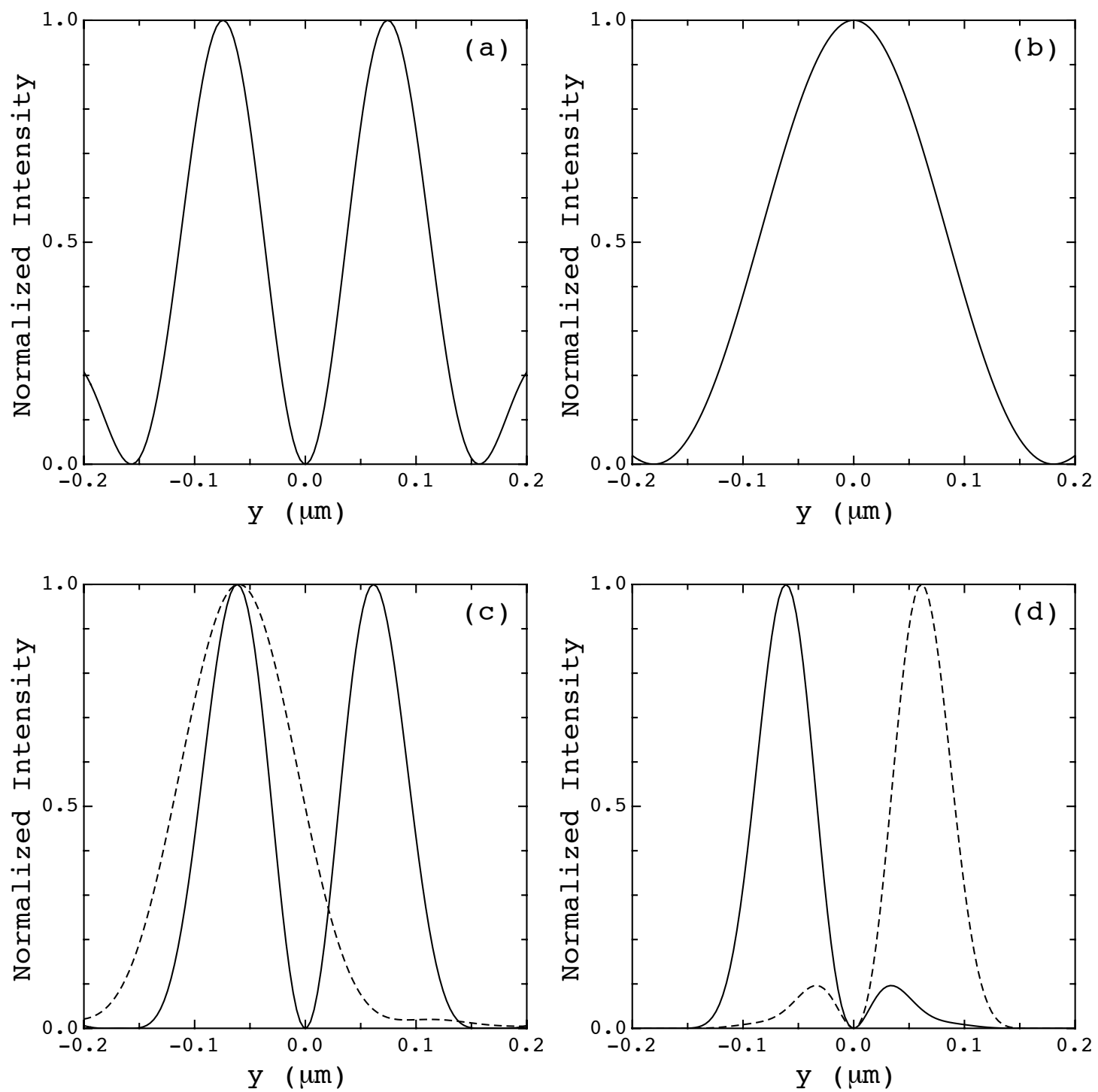

O. Haeberlé, "Improving the lateral resolution

. Figure 8 